\title{
An intelligent LED landscape lighting system
}

\author{
Lei Zhang ${ }^{1,2, *}$, Jie Fang ${ }^{1,2}$ and Ping $\mathrm{Yu}^{1,2}$ \\ ${ }^{1}$ School of electrical and photoelectrical Engineering. West Anhui University, Lu'an, Anhui, 237012 \\ ${ }^{2}$ Intelligent Lighting and Display Technology Engineering center, West Anhui University, Lu'an, \\ Anhui, 237012
}

\begin{abstract}
This paper introduces a kind of landscape lighting control system is composed of the monitoring center and intelligent controller. The intelligent controller realizes the control of the LED landscape lamp, and the monitoring center realizes the monitoring management of the system. The monitoring center and the intelligent controller communicate with each other through ZigBee net. This distributed LED landscape lighting system based on ZigBee network, can achieve the dimming, equipment monitoring, mode setting and fault alarm function, can effective management of landscape lighting system is composed of a large number of LED, solve the problems of large landscape lighting system lighting effects, monotonous monitoring management function is not perfect, the realization of LED landscape lighting system is difficult large scale problem.
\end{abstract}

\section{Introduction}

With the development of city and the improvement of people's living standard, more and more applications of various types of environmental lighting landscape lighting in the city, become a propaganda city, an important infrastructure to beautify the environment. Landscape lighting not only to illuminate the ground, creating a pleasant and comfortable atmosphere, but also pay attention to environmental protection and energy saving. Landscape lighting control system can fully reflect the advantages of LED used in landscape lighting, the color performance, strong light and color changing characteristics of LED with the most incisive play out, with appropriate control strategies, in accordance with the overall requirements of the environment, the programmed control of LED, through the LED color coordination, can produce the overall art the effect of landscape, showing a gorgeous scene. As landscape lighting facilities are mostly outdoors, the landscape control system combining intelligent control and monitoring is more in line with the needs of the modern people.

\footnotetext{
*Lei Zhang: leizh0326@wxc.edu.cn
} 


\section{LED landscape lighting system overall design}

\subsection{System composition}

The system adopts the distributed system structure, and the system structure diagram is shown in Figure 1. It consists of the control center, the ZigBee gateway and the distributed controller.

The wireless communication is realized between the control center and the controller through the ZigBee gateway. The system uses PC as the control center, and the administrator can realize the remote monitoring and management of the equipment by operating the $\mathrm{PC}$ the control software. The controller receives the command of the control center through the ZigBee network, performs the operation according to the command request, realizes the pattern dimming and supplies the equipment information back to the monitoring center.

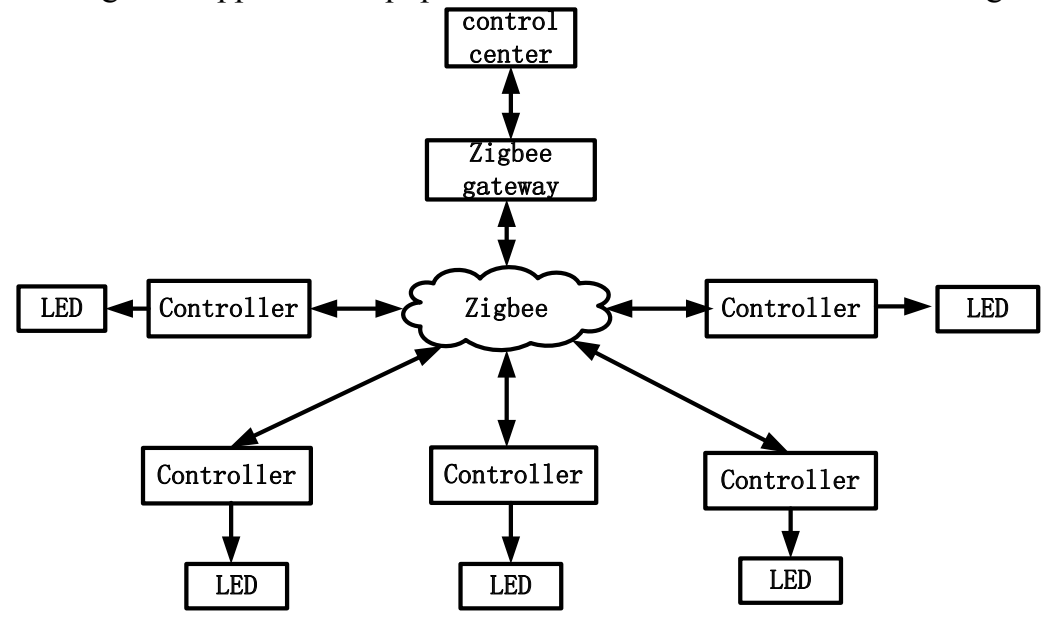

Fig. 1. System Structure Diagram

\subsection{Function of Each Part}

\subsubsection{The functions of the control computer}

(1) Provide human-computer interface for remote management and control

Through the control computer, a region LED lamps or a certain LED lamp can be controlled;

(2) Data monitoring and statistics

The control computer displays the running state of the LED device (real time current, voltage, etc.) in real time, collects and processes all the LED running data, and stores the data in the database server;

(3) Fault information management

When the LED lamp fails, an alarm signal is generated to facilitate the timely, accurate and convenient detection of the fault LED lamp, and the failure information is managed and monitored;

(4) Energy-saving control

LED landscape lighting system can automatically switch light and light according to the preset time or the time of sunrise and sunset. 


\subsubsection{ZigBee gateway}

It is composed of two modules, ZigBee coordinator and serial communication, which is the communication hub of the system. ZigBee is an emerging wireless network technology, mainly used for short-range wireless network connections. It uses IEEE802.15.4 as standard to communicate between a large number of tiny sensors. These sensors require very low energy to relay data from one sensor to another in a relay mode to achieve efficient communication. ZigBee network has many advantages, such as low power consumption, low cost, high capacity (up to 65536 nodes), and it can solve the difficult problems of implementation and construction quickly and conveniently. Also use $2.4 \mathrm{GHz}$ free wireless frequency without charge for use. The serial communication module adopts RS232/485 standard interface technology, which can effectively solve the problem of wireless data communication and wired data communication network access.

\subsubsection{The controller}

As the terminal control equipment of the system, the dimmer is connected with the LED lamp and its main function.

(1) Detecting LED lamp input voltage, current, temperature and other operating conditions;

(2) According to the control commands sent by the monitoring center, the operation is carried out, and the functions of lighting adjustment, equipment and lighting information feedback are realized;

(3) Controlling the LED switch and brightness regulation.

\subsubsection{LED lamp}

The RGB LED, which is packaged as 5050, is built with opposing red LED (R), green LED (G) and blue LED (B). R, G, B produces red, green, blue three colors, for RGB LED, realizes each kind of color to provide the foundation. Among them, the red LED power supply range is $2.2 \mathrm{~V} \sim 2.6 \mathrm{~V}$, green LED power supply range is $3.2 \mathrm{~V} \sim 3.6 \mathrm{~V}$, blue LED power supply range is $3.2 \mathrm{~V} \sim 3.6 \mathrm{~V}$. Three built-in LED operating currents are $25 \mathrm{~mA}$.

\section{Hardware design of led landscare lighting controller}

The controller is composed of C8051F020 microcontroller, serial clock chip DS1307, LED driver PT4115, ZigBee communication module CC2530 and LED detection circuit. The block diagram is shown in figure 2.

\subsection{C8051F020 microcontroller}

C8051F020 microcontroller reads the clock, according to the time to achieve the LED landscape lighting on and off. At the same time receive the host computer through the ZigBee network instructions, to achieve the LED switch and brightness adjustment. If the host does not have instructions, it opens the landscape lighting in default mode, detects the status of LED in the LED lighting state, implements LED protection, and reports the LED status through the ZigBee network. 


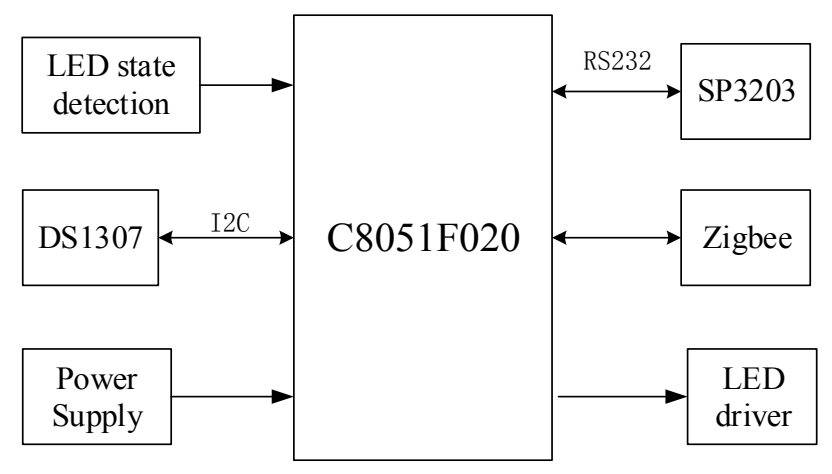

Fig. 2. Controller Structure Diagram

\subsection{LED status detection circuit}

1. Temperature detection circuit

LED has a negative temperature effect, positive current increases will cause a rise in junction temperature, temperature rise and further lead to the current increases, when the temperature exceeds LED bearing temperature will make the LED luminous efficiency is reduced, causing LED damage. Therefore, the system should have LED temperature detection function. To prevent the LED temperature is too high, to prevent decay, prolong the service life of the lamp. The digital temperature sensor DS18B20 is directly contacted with the LED board to measure the working temperature of the LED.

The light detecting circuit is used for detecting whether the LED works normally, and the light detecting circuit adopts a photosensitive diode, and the photosensitive junction of the photosensitive diode has a photosensitive property, and has a single guiding electric property.

2. Illumination detection circuit

The light detecting circuit is used for detecting whether the LED works normally. The light detecting circuit adopts a photosensitive diode, and the photosensitive junction of the photosensitive diode has a photosensitive property, and has a single guiding electric property. If the photodiode does not accept the light, the reverse leakage current is smaller, the circuit is not switched on, and when the circuit receives the light, the current is greatly increased, and the circuit is turned on. Because the current system of LED lighting needs is relatively low, only 30mA, the current sensor must use high precision, using ACS712 linear current sensor of Allergro company, built-in high precision linear hall sensor circuit, voltage current detection which can be loaded and the corresponding voltage value, and then read the current conversion of microprocessor through the A/D.

\subsection{Dimming isolation circuit}

In order to avoid the influence of LED constant current drive circuit on the control circuit, the photoelectric isolation method is used to isolate the strong current and the weak current. The PWM signal of the microcontroller is sent to the dimming pin of the PT4115 driver chip through the photoelectric isolator PC817. When the PWM is high, the driver chip works normally and the LED flows through the rated current. When the PWM is low, the LED driver cuts off the current on the LED light string. The brightness adjustment of LED is realized by adjusting the duty ratio of PWM. 


\subsection{LED drive circuit}

According to the host computer instruction or the default mode, the MCU generates three PWM signals, controls the LED driver PT4115 current output, and controls the three color LED brightness of LED, G, B and R respectively, and realizes the color control of LED.

\section{Software design of control system}

\subsection{Gateway program design}

Because the ZigBee protocol stack is a semi open source protocol stack, the protocol stack architecture provided by TI company has written the physical layer, media access layer, network layer and the underlying code, as long as the realization of communication protocol module can meet the corresponding.

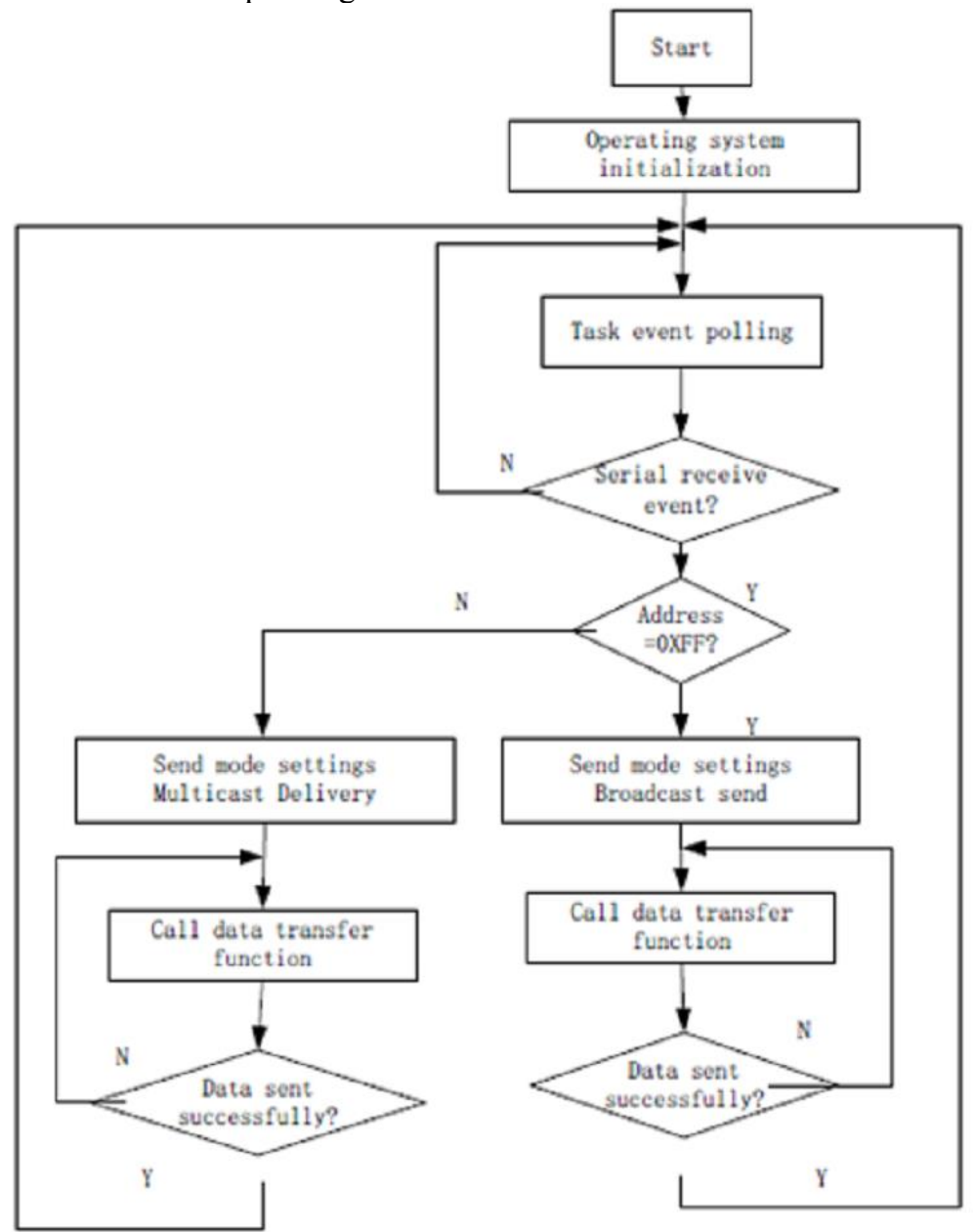

\section{Fig. 3. Gateway Flowchart}

The ZigBee gateway application flow diagram is shown in figure 3. After the ZigBee module is powered on, the system is initialized first, then the network is set up and started, and the system events are polled after the network is successfully set up. This paper is mainly 
applied to join the gateway node network, serial data reception event poll on whether, if a serial data reception, call the serial receiver function, remove the address information and control information is transmitted to the PC to read from the register, and to estimate the address information, if the address is $0 \mathrm{xFF}$, then the transmission mode is set to broadcast, and sends control information to the ZigBee network in the all terminal lamp node; if the address is not $0 \mathrm{xFF}$, it will send the multicast mode to send, and sends control information to the designated terminal lights all nodes within the group. In order to reduce the packet loss rate of data, the data sent out should also confirm whether the data is sent successfully, if not sent successfully, you need to resend.

\subsection{Program design of controller node}

After the control node device is powered on, the ZigBee module should first apply to join the network after initialization, and then access the operating system polling after obtaining the network access from the ZigBee gateway. The terminal equipment is mainly to check whether the data receiving event, if the data is received, then call the data receiving function, and will receive the data assigned to the PWM module register, output PWM dimming signal waveform, the data is successfully received at the same time, the terminal node sending data to coordinator should also receive success in order to inform the signal, the coordinator has successfully received data; if no data is received, according to the default mode controller PWM control LED light. 


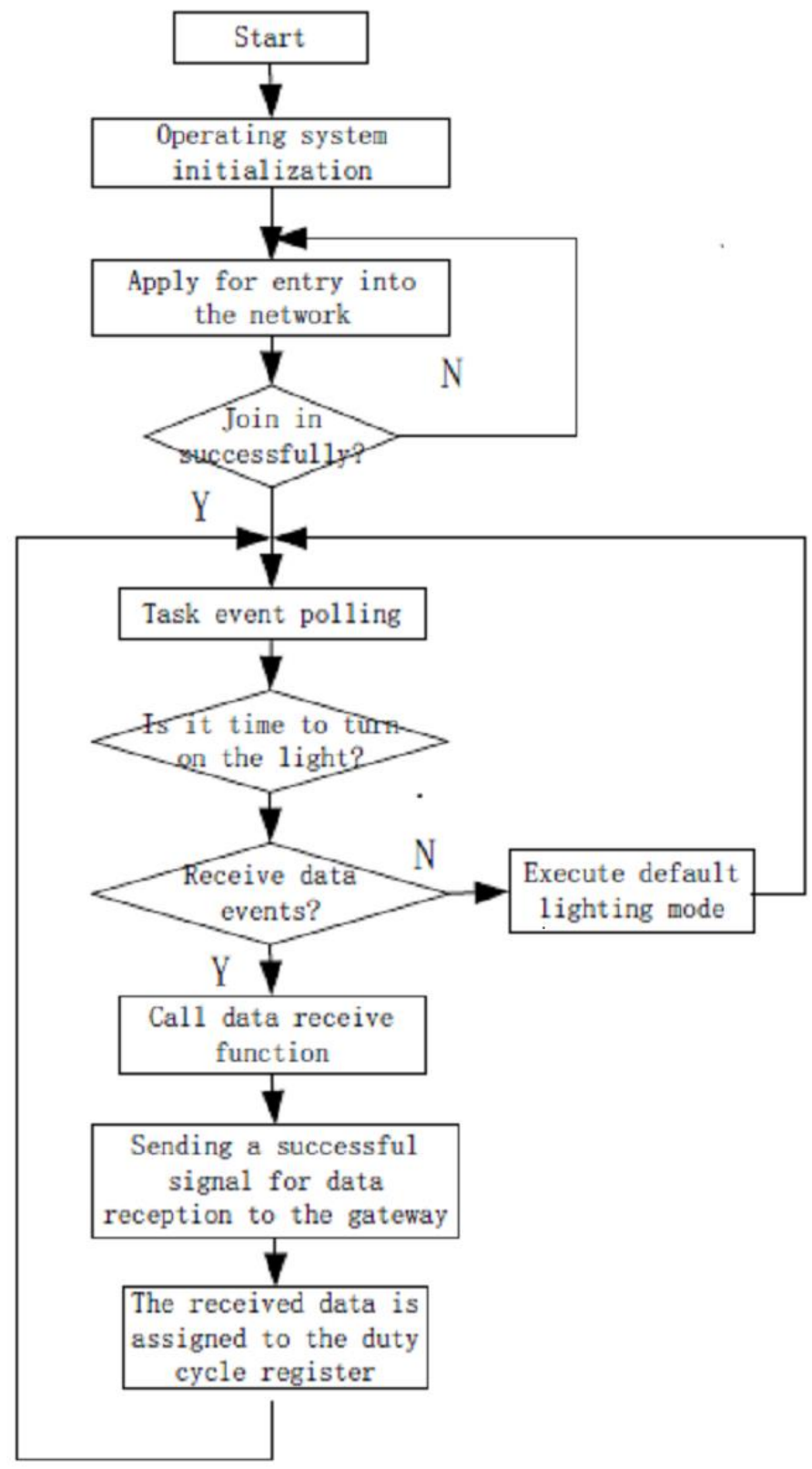

Fig. 4. Flow chart of controller program

\subsection{Control center program}

Intelligent landscape lamp control system, the upper computer interface uses Forcecontrol 7.1 development, mainly has control draw, monitoring draw, alarm draw, reports draw. switch light control, mode selection, area switch control and single light switch control. Control draw, switch light control, mode selection, area switch control and single light switch control. The monitoring draw monitors the status of the landscape lamp in real time, and 
displays the switch status, current, voltage, mode, etc. of the landscape lamp. Alarm draw to achieve fault alarm, when the current is too large, too large voltage, high temperature alarm.

\section{Conclusion}

In this paper, designed a LED landscape lighting control system based on configuration software and ZigBee network, introduces the overall architecture of the system and the working principle and realization method of hardware circuit and software, and verified by experiment. The system can provide various control functions and real-time monitoring functions for LED through wireless network communication on the PC, so as to meet the needs of different landscape lighting. To some extent, the problems such as monotonous lighting effects, imperfect monitoring and management functions and great difficulty in the expansion of large-scale landscape lighting systems are solved.

This work is supported by NFSC61302179, WXZR201620.

\section{References}

[1] Hangfeng Ji, Qiong Zhao, Xiaodong Wang, et al. Zig Bee-based LED intelligent lighting control system// 10th China International Forum on Solid State Lighting (China SSL). 2013.

[2] Faheem Ijaz, Adeel A. Siddiqui, Byung Kwan Im, Chankil Lee. Remote management and control system for LED based Plant Factory using ZigBee and Internet // 14th International Conference on Advanced Communication Technology (ICACT). 2012:942-946.

[3] He Sai, Chen Xiaoping. Application of Zig Bee technology in urban lighting monitoring system [J]. Computer system applications, 2011, 20(11):135-138.

[4] CaiYu. The Design and Implementation of LED Landscape Lighting Control System. Zhejiang University. 2015.

[5] Li Zhihua, Zhou Feng, Wang Yuanzhang, Yan Gang. LED intelligent lighting control system based on ZigBee. Modern building electrical. No. 6.Vol4.9-13. 